\title{
An Improved Nonlinear Grey Bernoulli Model Combined with Fourier Series
}

\author{
Wang Chia-Nan and Phan Van-Thanh \\ Department of Industrial Engineering and Management, National Kaohsiung University of Applied Sciences, \\ 415 Chien-Kung Road, Kaohsiung 807, Taiwan
}

Correspondence should be addressed to Phan Van-Thanh; thanhkem2710@gmail.com

Received 28 April 2015; Revised 3 August 2015; Accepted 12 August 2015

Academic Editor: Meng Du

Copyright (c) 2015 W. Chia-Nan and P. Van-Thanh. This is an open access article distributed under the Creative Commons Attribution License, which permits unrestricted use, distribution, and reproduction in any medium, provided the original work is properly cited.

\begin{abstract}
Grey forecasting is a dynamic forecasting model and has been widely used in various fields. In recent years, many scholars have proposed new procedures or new models to improve the precision accuracy of grey forecasting for the fluctuating data sets. However, the prediction accuracy of the grey forecasting models existing may not be always satisfactory in different scenario. For example, the data are highly fluctuating are with lots of noise. In order to deal with this issue, a Fourier Nonlinear Grey Bernoulli Model $(1,1)$ (abbreviated as F-NGBM $(1,1)$ ) is proposed to enhance the forecasting performance. The proposed model was established by using Fourier series to modify the residual errors of Nonlinear Grey Bernoulli Model $(1,1)$ (abbreviated as (NGBM $(1,1)$ ). To verify the effectiveness of the proposed model, fluctuation data of the numerical example in Wang et al.s paper (Wang et al. 2011) and practical application are used. Both of these simulation results demonstrate that the proposed model could forecast more precisely than several different kinds of grey forecasting models. For future direction, this proposed model can be applied to forecast the performance with the high fluctuation data in the different industries.
\end{abstract}

\section{Introduction}

Grey forecasting is the main part of grey system theory and an effective method for modeling and forecasting small sample time series. In the early 1980 s, Professor Deng $[1,2]$ proposed the grey model $(\mathrm{GM})(1,1)$ based on control theory. This model utilizes an operator obtained by the first-order accumulation to operate the nonnegative original sequence. It demonstrates the approximate exponential growth laws and achieves short-term forecasting accuracy. With its advantages in dealing with uncertain information and few data required [3-5], the GM $(1,1)$ has been widely and successfully applied to various fields such as tourism $[6,7]$, transportation [8-10], financial and economic [11-13], integrated circuit industry [14-17], and energy industry [18-20].

In the recent years, many scholars have proposed new procedures or new models to improve the precision accuracy of grey model. For instant, Lin et al. [21] and Wang et al. [22] used different methods to improve the background values. Hsu [17] and Wang and Hsu [23] used different methods to modify the internal parameter estimation, like development coefficient and grey input coefficient. Some scholars had established GM $(1,1)$ model with residuals modification $[15$, 24]. In addition, many hybrid models based on $\operatorname{GM}(1,1)$ were proposed. These included the grey econometric model [25], the grey Markov model $[26,27]$, and the grey fuzzy model [21]. Despite its improvement in prediction accuracy, the prediction accuracy of the GM $(1,1)$ model is always monotonic. As a result, GM $(1,1)$ model may not be always satisfactory.

The recently developed, Nonlinear Grey Bernoulli Model (NGBM $(1,1)$ ) was named by Chen $[28,29]$ and firstly appeared in the book [30]. The NGBM $(1,1)$ has greater flexibility than GM $(1,1)$ and Grey-Verhulst model by adjusting power index. Therefore, forecasting of the fluctuation sequence can be performed, as long as the power exponent and structural parameters in the model are known. Because of the flexibility of NGBM $(1,1)$ model, it had a great variety of application to simulate and forecast in different fields. Chen [28] proposed the NGBM $(1,1)$ to forecast 
the annual unemployment rates of ten selected countries to help governments to develop future policies regarding labor and economic policies. At the same time, Chen et al. [29] also used $\operatorname{NGBM}(1,1)$ to forecast foreign exchange rates of twelve Taiwan major trading partners in 2005. Both of the two above studies indicated that the $\operatorname{NGBM}(1,1)$ can improve the accuracy of the simulation and forecasting predictions of the original GM $(1,1)$.

Some scholars had tried to improve the $\operatorname{NGBM}(1,1)$ from different aspects recently, such as Zhou et al. [31] who selected the parameter value of $n$ by using a particle swarm optimization algorithm and used the model to forecast the power load of the Hubei electric power network. Hsu [16] used the genetic algorithm to optimize the parameters of the $\operatorname{NGBM}(1,1)$ and applied it to forecast the economic trends in the integrated circuit industries in Taiwan. Chen et al. [32] proposed a Nash NGBM $(1,1)$ based on the Nash equilibrium concept. This strengthens the adaptability of the model and eventually improves the accuracy of the model. Later, Wang et al. [33] proposed optimized NGBM $(1,1)$ model to forecast the qualified discharge rate of the industrial waste water in 31 administrative areas in China by improved background interpolation value $p$ and exponential value $n$. Wang [34] proposed the optimized Nash $\operatorname{NGBM}(1,1)$ by optimizing the initial conditions to forecast the main economic indices of high technology enterprises in China. Performance evaluation of this results showed that the optimized model can fit the data well and provide guidance for policy making decisions for the development of high technology enterprise and so on.

Although those improved $\operatorname{NGBM}(1,1)$ models have been successfully adopted in various fields and they have provided us with promising results, the $\operatorname{NGBM}(1,1)$ is not always satisfactory in some special scenarios. For example, the data are highly fluctuating or are with lots of noise. In order to deal with these issues, this paper based on the advantages of Nonlinear Grey Bernoulli Model $(1,1)$ and Fourier series to build an effectiveness model aims to increase the predictive accuracy. The proposed model is a two-stage procedure; the first stage is using the $\operatorname{NGBM}(1,1)$ to get the predicted value and then using Fourier series to modify the residual errors of $\operatorname{NGBM}(1,1)$. The Fourier series transform the residuals error of $\operatorname{NGBM}(1,1)$ into frequency spectra, and then the researchers select the low-frequency term. This way can filter out high-frequency terms, which are supposed to be noisy, and then have better performance. To verify the effectiveness of the proposed model, both fluctuation data of the numerical example in Wang et al.s paper [33] and practical application are used. All these simulation results indicated that the proposed model could offer a more precise forecast than several different kinds of grey forecasting models. Through simulation results, this study offers an effective model in order to deal with the high fluctuation sequence.

The remainder of this paper is organized as follows. Section 2 briefly introduces the original $\operatorname{NGBM}(1,1)$ and the F-NGBM $(1,1)$. Section 3 demonstrates that F-NGBM $(1,1)$ has better performances in several numerical examples by comparison with optimized $\operatorname{NGBM}(1,1)$, original NGBM $(1,1)$, optimized GM $(1,1)$, and the original $\operatorname{GM}(1,1)$. Finally, the conclusions are made in Section 4.

\section{Methodology}

2.1. A Brief Introduction to the Nonlinear Grey Bernoulli Model. The Nonlinear Grey Bernoulli Model (NGBM) $(1,1)$ is a first-order single-variable grey Bernoulli model with an interpolated coefficient in the background value [28, 29]. According to Zhou et al. [31], the procedures involved in using the $\operatorname{NGBM}(1,1)$ can be summarized as follows.

Step 1. Let raw matrix $X^{(0)}$ stand for the nonnegative original historical time series data

$$
X^{(0)}=\left\{x^{(0)}\left(t_{i}\right)\right\}, \quad i=1,2, \ldots, m,
$$

where $x^{(0)}\left(t_{i}\right)$ corresponds to the system output at time $t_{i}$ and $m$ is the total number of modeling data.

Step 2. Construct $X^{(1)}$ by one time accumulated generating operation (1-AGO), which is

$$
X^{(1)}=\left\{x^{(1)}\left(t_{i}\right)\right\}, \quad i=1,2, \ldots, m,
$$

where $x^{1}\left(t_{k}\right)=\sum_{i=1}^{k} x^{(0)}\left(t_{i}\right), k=1,2, \ldots, m$.

Step 3. The grey differential equation of $\operatorname{NGBM}(1,1)$ is defined as

$$
x^{(0)}\left(t_{k}\right)+a z^{(1)}\left(t_{k}\right)=b\left[z^{(1)}\left(t_{k}\right)\right]^{n} .
$$

And its whitenization differential equation is as follows:

$$
\frac{d x^{(1)}\left(t_{k}\right)}{d t_{k}}+a x^{(1)}\left(t_{k}\right)=b\left[x^{(1)}\left(t_{k}\right)\right]^{n},
$$

where $z^{(1)}\left(t_{k}\right)=p x^{(1)}\left(t_{k}\right)+(1-p) x^{(1)}\left(t_{k-1}\right), k=2,3, \ldots, m$; $p$ is called the production coefficient of the background value with a close interval $[0,1]$, which is traditionally set to 0.5 .

The parameters $a, b$ and the power of $n$ are called the developing coefficient, the named grey input, and an adjustable parameter, respectively, for the power of " $n$ " belonging to any real number excluding $n=1$.

Step 4. From (4), the value of parameters $a$ and $b$ can be estimated by using ordinary least-square method (OLS). That is,

$$
\left[\begin{array}{l}
a \\
b
\end{array}\right]=\left(B^{T} B\right)^{-1} B^{T} Y_{m}
$$

where

$$
\begin{aligned}
B & =\left[\begin{array}{cc}
-z^{(1)}\left(t_{2}\right) & \left(z^{(1)}\left(t_{2}\right)\right)^{n} \\
-z^{(1)}\left(t_{3}\right) & \left(z^{(1)}\left(t_{3}\right)\right)^{n} \\
\ldots & \ldots \\
-z^{(1)}\left(t_{m}\right) & \left(z^{(1)}\left(t_{m}\right)\right)^{n}
\end{array}\right], \\
Y_{m} & =\left[\begin{array}{c}
x^{(0)}\left(t_{2}\right) \\
x^{(0)}\left(t_{3}\right) \\
\cdots \\
x^{(0)}\left(t_{m}\right)
\end{array}\right] .
\end{aligned}
$$


Step 5. The solution of (4) can be obtained after the parameters $a$ and $b$ have been estimated. That is,

$$
\begin{array}{r}
\widehat{x}^{(1)}\left(t_{k}\right) \\
=\left[\left(x^{(0)}\left(t_{1}\right)^{(1-n)}-\frac{b}{a}\right) e^{-a(1-n)\left(t_{k}-t_{1}\right)}+\frac{b}{a}\right]^{1 /(1-n)}, \\
n \neq 1, k=1,2,3, \ldots
\end{array}
$$

Step 6. Applying inverse accumulated generating operation (I-AGO) to $\widehat{x}^{(1)}\left(t_{k}\right)$, the predicted data of $\widehat{x}^{(0)}\left(t_{k}\right)$ can be estimated as

$$
\begin{aligned}
& \hat{x}^{(0)}\left(t_{k}\right) \\
& =\left\{\begin{array}{l}
\hat{x}^{(0)}\left(t_{k}\right)=x^{(0)}\left(t_{1}\right) \\
\hat{x}^{(0)}\left(t_{k}\right)=\widehat{x}^{(1)}\left(t_{k}\right)-\hat{x}^{(1)}\left(t_{k-1}\right) \quad(k=2,3, \ldots, m) .
\end{array}\right.
\end{aligned}
$$

2.2. The Residual of $\operatorname{NGBM}(1,1)$ Modification by Fourier Series. Because Fourier series can transform the residuals error into frequency spectra and then select the lowfrequency terms, moreover, Fourier technique can filter out high-frequency terms, which are supported to be noise, and then have better performance. Therefore, this study uses the Fourier series [6] to modify the residual of the $\operatorname{NGBM}(1,1)$ for improving the prediction accuracy. The overall procedure to obtain the modified model is as follows.

Let $x$ be the original series of $m$ entries and $v$ is the predicted series (obtained from $\operatorname{NGBM}(1,1)$ ). Based on the predicted series $v$, a residual series named $\varepsilon$ is defined as

$$
\varepsilon=\{\varepsilon(k)\}, \quad k=2,3, \ldots, m,
$$

where

$$
\varepsilon(k)=x(k)-v(k), \quad k=2,3, \ldots, m .
$$

According to the definition of the Fourier series, the residual sequence of $\operatorname{NGBM}(1,1)$ can be approximately expressed as

$$
\begin{aligned}
& \widehat{\varepsilon}(k) \\
&=\frac{1}{2} a_{(0)} \\
&+\sum_{i=1}^{Z}\left[a_{i} \cos \left(\frac{2 \pi i}{m-1}(k)\right)+b_{i} \sin \left(\frac{2 \pi i}{m-1}(k)\right)\right], \\
& k=1,2,3, \ldots, m,
\end{aligned}
$$

where $Z=((m-1) / 2)-1$ is called the minimum deployment frequency of Fourier series $[6,35]$ and $Z$ only be taken integer number.

Therefore, the residual series is rewritten as

$$
\varepsilon=P C,
$$

where

The parameters $a_{0}, a_{1}, b_{1}, a_{2}, b_{2}, \ldots, a_{Z}, b_{Z}$ are obtained by using the ordinary least squares (OLS) method whose results are in the following equation:

$$
C=\left(P^{T} P\right)^{-1} P^{T} \varepsilon^{T} .
$$

Once the parameters are calculated, the modified residual series is then achieved based on the following expression:

$$
\begin{aligned}
\widehat{\mathcal{E}}(k) & \\
= & \frac{1}{2} a_{0} \\
& +\sum_{i=1}^{Z}\left[a_{i} \cos \left(\frac{2 \pi i}{m-1}(k)\right)+b_{i} \sin \left(\frac{2 \pi i}{m-1}(k)\right)\right] .
\end{aligned}
$$

From the predicted series $v$ and $\widehat{\varepsilon}$, the Fourier modified series $\widehat{v}$ of series $v$ is determined by

$$
\widehat{v}=\left\{\widehat{v}_{1}, \widehat{v}_{2}, \widehat{v}_{3}, \ldots, \widehat{v}_{k}, \ldots, \widehat{v}_{m}\right\}
$$

where

$$
\widehat{v}=\left\{\begin{array}{l}
\widehat{v}_{1}=v_{1} \\
\widehat{v}_{k}=v_{k}+\widehat{\varepsilon}_{k} \quad(k=2,3, \ldots, m) .
\end{array}\right.
$$

2.3. Evaluative Precision of Forecasting Models. In order to evaluate the forecast capability of the model, Means Absolute 
TABLE 1: Forecasted results from the grey models.

\begin{tabular}{|c|c|c|c|c|c|c|c|c|c|c|c|}
\hline \multicolumn{2}{|c|}{ Original value } & \multicolumn{2}{|c|}{ GM $(1,1)$} & \multicolumn{2}{|c|}{$\begin{array}{c}\text { Optimized GM }(1,1) \\
p=0.99\end{array}$} & \multicolumn{2}{|c|}{$\begin{array}{c}\text { Original NGBM }(1,1) \\
n=-10, p=0.5\end{array}$} & \multicolumn{2}{|c|}{$\begin{array}{r}\text { Optimized NGBM }(1,1) \\
n=-19.58, p=0.569\end{array}$} & \multicolumn{2}{|c|}{$\begin{array}{l}\text { F-NGBM }(1,1) \\
n=-10, p=0.5\end{array}$} \\
\hline & $x^{(0)}(k)$ & $\hat{x}^{(0)}(k)$ & MAPE (\%) & $\widehat{x}^{(0)}(k)$ & MAPE (\%) & $\hat{x}^{(0)}(k)$ & MAPE (\%) & $\widehat{x}^{(0)}(k)$ & MAPE (\%) & $\widehat{x}^{(0)}(k)$ & MAPE (\%) \\
\hline$k=1$ & 5 & 5 & 0 & 5 & 0 & 5 & 0 & 5 & 0.1 & 5 & 0 \\
\hline$k=2$ & 6 & 5.084 & 15.26 & 5.488 & 8.527 & 6.499 & 8.31 & 6.00 & 0.00 & 6 & 0 \\
\hline$k=3$ & 4 & 5.634 & 40.86 & 5.866 & 46.66 & 4.921 & 23.03 & 4.828 & 20.70 & 4 & 0 \\
\hline$k=4$ & 7 & 6.920 & 10.80 & 6.271 & 10.42 & 6.986 & 0.2 & 6.946 & 0.77 & 7 & $\mathbf{0}$ \\
\hline MAPE (\% & & & 16.73 & & 16.42 & & 7.89 & & 7.16 & & 0.00 \\
\hline
\end{tabular}

Percentage Error (MAPE) index is used in this study to evaluate the performance and reliability of forecasting technique [36]. It is expressed as follows:

$$
\text { MAPE }=\frac{1}{m} \sum_{k=2}^{m}\left|\frac{x^{(0)}(k)-\hat{x}^{(0)}(k)}{x^{(0)}(k)}\right| \times 100 \%,
$$

where $x^{(0)}(k)$ and $\widehat{x}^{(0)}(k)$ are actual and forecasting values in time period $k$, respectively, and $m$ is the total number of predictions.

Wang and Phan [35] interpret the MAPE results as a method to judge the accuracy of forecasts, where more than $10 \%$ is an inaccurate forecast, $5 \%-10 \%$ is a reasonable forecast, $1 \%-5 \%$ is a good forecast, and less than $1 \%$ is an excellent forecast.

\section{Validation of the F-NGBM $(1,1)$}

In this section, two examples are given to compare the proposed model with several different kinds of grey forecasting models, which are the optimized $\operatorname{NGBM}(1,1)[30,33]$, original NGBM $(1,1)$ [28], optimized GM $(1,1)[6]$, and original GM $(1,1)[6,8]$, to show the effectiveness of proposed model in the high fluctuation data sets. The first example in this study is proposed in Wang' paper [33] and the second example is the real case study of the gold price (GP) in the afternoon from the London Fix.

The procedures of the optimized NGBM $(1,1)$ and optimized GM $(1,1)$ models were established by minimizing an objective function of (18) with the constraints being $0 \leq p \leq 1$ and $n \neq 1$ to get the global optimization of parameters $p$ and $n$ (just for $\operatorname{NGBM}(1,1)$ ). More detailed procedures of these models were comprehensively illustrated in Chen et al. [29] and Wang et al. [33]. In terms of the proposed prediction model, the procedure has two stages. The first stage is to build the $\operatorname{NGBM}(1,1)$ to roughly predict the next data from a set of the most recent data. The second stage is to use Fourier series to refine the residual error by the $\operatorname{NGBM}(1,1)$. More details are given below.

3.1. Fluctuating Raw Data Sequence Example. First example, F-NGBM $(1,1)$ is used to predict an example proposed in the Wang et al's paper [33]. In this example, the raw data sequence jumps randomly $X^{(0)}=(5,6,4,7)$ in this case. Wang et al. used as an example to demonstrate the improvement in the accuracy of the optimized NGBM $(1,1)$. In this section, we

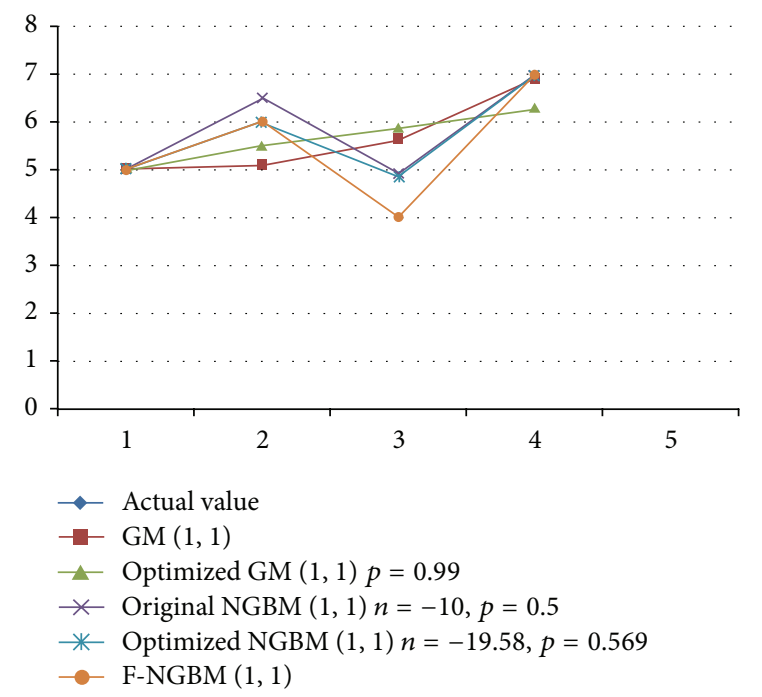

Figure 1: Original fluctuation sequence curves versus forecasts.

also adopt this example to compare the forecasting performance of the F-NGBM $(1,1)$ with the optimized $\operatorname{NGBM}(1,1)$ and the original NGBM $(1,1)$ in Wang et al. [33]. Forecasting results are shown in Table 1 and Figure 1.

Table 1 reveals that the optimized $\operatorname{NGBM}(1,1)$ with $n=$ -19.58 and $p=0.569$ has a higher accuracy than the original $\operatorname{NGBM}(1,1)$ with $n=-10$ and $p=0.5$ as well as the optimized GM $(1,1)$ and original GM $(1,1)$. By adopting the F-NGBM $(1,1)$ with parameters $n=-10$ and $p=0.5$, the MAPE of the F-NGBM $(1,1)$ decreased from $7.16 \%$ to $0 \%$. This result clearly indicated that the F-NGBM $(1,1)$ is the best fitting model among five forecasting models on the same sequence. Much clearer visualization is showed in Figure 1.

3.2. The Gold Price Forecasting. To give more evidence about the forecasting ability of the proposed model, this paper uses the real case in the life. This study uses the historical data of gold price (GP) from the London PM Fix, from day 03/11/2014 to $28 / 4 / 2015$, to verify the effectiveness of F-NGBM $(1,1)$. The data of gold price is obtained from the daily statistical data published on the website of Kitco [37]. There are totally 122 observations available as illustrated in Figure 2. From Figure 2, the trend of gold price (GP) is a wild fluctuation and is highly nonlinear over the period. To demonstrate the superiority of proposed model for both of interpolation and 
TABLE 2: The parameter evaluation of the fitting models.

\begin{tabular}{lc}
\hline Model & Parameter value \\
\hline GM $(1,1)$ & $n=0, p=0.5$ \\
Optimized GM $(1,1)$ & $n=0, p=0.001$ \\
Original NGBM $(1,1)$ & $n=0.0381, p=0.5$ \\
Optimized NGBM $(1,1)$ & $n=0.0385, p=0.001$ \\
F-NGBM $(1,1)$ & $n=0.0381, p=0.5$ \\
\hline
\end{tabular}

TABLE 3: In-sample comparisons among five grey forecasting models.

\begin{tabular}{lccc}
\hline Model & MAPE (\%) & $\begin{array}{c}\text { Forecasted } \\
\text { accuracy }(\%)\end{array}$ & Performance \\
\hline GM $(1,1)$ & 2.1113 & 97.8887 & Good \\
Optimized GM $(1,1)$ & 2.1108 & 97.8892 & Good \\
Original NGBM $(1,1)$ & 1.7948 & 98.2052 & Good \\
Optimized NGBM $(1,1)$ & 1.7791 & 98.2209 & Good \\
F-NGBM $(1,1)$ & $\mathbf{0 . 0 0 0 8}$ & $\mathbf{9 9 . 9 9 9 2}$ & Excellent \\
\hline
\end{tabular}

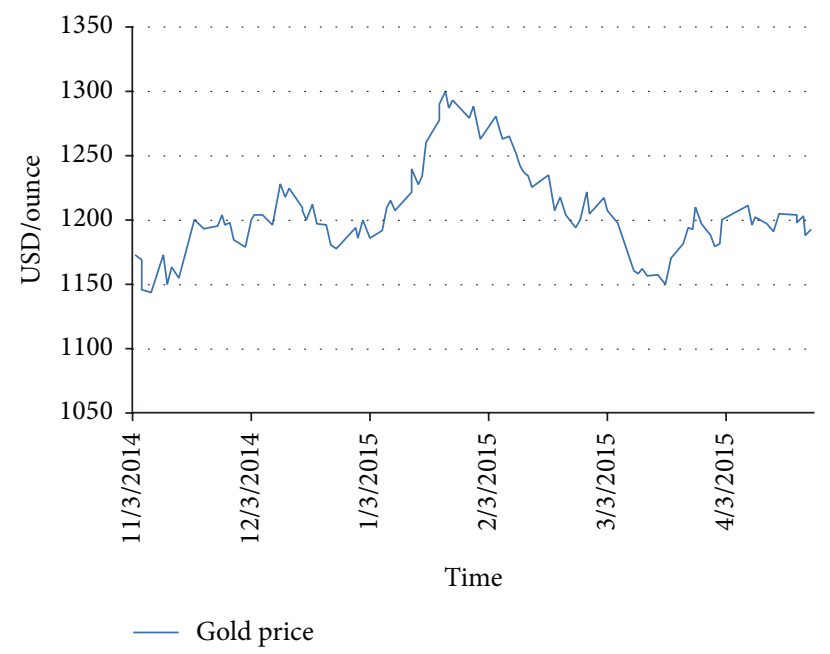

Figure 2: Gold price time series from the day 03/11/2014 to 28/4/2015.

extrapolation data, this study sets the samples from 03.11.2004 to 24.04.2015 (120 data points) for in-sample estimation. And the remainders of the sample are reserved for out-of-sample forecasting purposes (or validation data set).

In order to find out the parameters in five forecasting models, which are original GM $(1,1)$, optimized $\operatorname{GM}(1,1)$, original $\operatorname{NGBM}(1,1)$, and optimized $\operatorname{NGBM}(1,1)$ as well as the proposed model, Microsoft Excel is used. Beside a basic function in Excel, Excel software also offers two useful functions named MMULT (array 1, array 2) to return the matrix product of two relevant arrays and Minverse (array) to return the inverse matrix. For the sake of convenience, the detailed calculation and modeling process are omitted here. All the parameters estimation results of these models are listed in Table 2. Only the MAPE of these models for both in-sample and out-of-sample forecasting are shown in Tables 3 and 4 , respectively.
TABLE 4: Out-of-sample comparisons among five grey forecasting models.

\begin{tabular}{lccc}
\hline Model & MAPE (\%) & $\begin{array}{c}\text { Forecasted } \\
\text { accuracy (\%) }\end{array}$ & Performance \\
\hline GM $(1,1)$ & 1.4475 & 98.5525 & Good \\
Optimized GM $(1,1)$ & 1.4450 & 98.5520 & Good \\
Original NGBM $(1,1)$ & 0.8074 & 99.1926 & Good \\
Optimized NGBM $(1,1)$ & 0.8073 & 99.1925 & Good \\
F-NGBM $(1,1)$ & $\mathbf{0 . 2 4 9 9}$ & $\mathbf{9 9 . 7 5 0 1}$ & Excellent \\
\hline
\end{tabular}

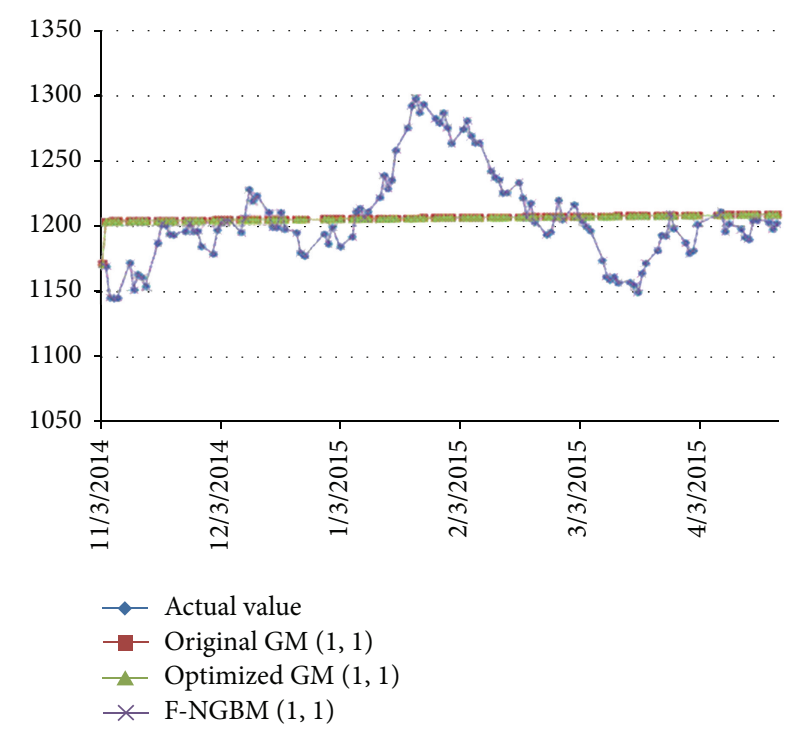

FIgURE 3: Curves of actual and simulated values using traditional and optimized GM $(1,1)$ models and F-NGBM $(1,1)$ for gold price forecasting.

Tables 3 and 4 show that the MAPE indexes of proposed model for in-sample and out-of-sample forecast are $0.0008 \%$ and $0.2499 \%$, respectively. These results indicate that the forecasted performance of proposed model is the best fitting performance among four forecasting models. In addition, Figures 3 and 4 emphasize that the curve of F-NGBM $(1,1)$ extremely closed with actual data than the curve of the remaining fours forecasting models. Similarly, the curves of optimized $\operatorname{NGBM}(1,1)$ and the original $\operatorname{NGBM}(1,1)$ have similar forecasting results (Figure 3 ) and the curves of optimized $\operatorname{GM}(1,1)$ and the original $\operatorname{GM}(1,1)$ are nearly the same (Figure 4).

\section{Conclusion}

In this paper, the effectiveness model named F-NGBM $(1,1)$ is proposed for improving the prediction accuracy in the high fluctuation data sets. The proposed prediction model approach uses the $\operatorname{NGBM}(1,1)$ to roughly predict the next data from a set of the most recent data and then uses the Fourier series to fit the residual error proceeded by the $\operatorname{NGBM}(1,1)$. From Tables 1,3 , and 4 , it is evident that F$\operatorname{NGBM}(1,1)$ could offer a better precise forecast than several 


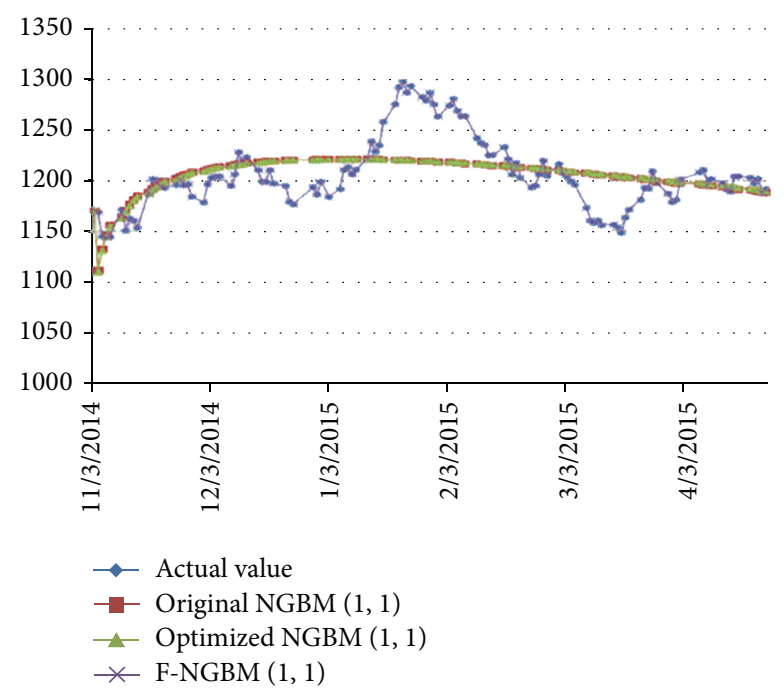

Figure 4: Curves of actual and simulated values using traditional and optimized NGBM $(1,1)$ and F-NGBM $(1,1)$ for gold price forecasting.

different kinds of grey forecasting models, such as optimized$\operatorname{NGBM}(1,1)$, original $\operatorname{NGBM}(1,1)$, optimized $\operatorname{GM}(1,1)$, and the original GM $(1,1)$.

Future researchers can be using different equations or different methodologies like the Markov Chain, neural network to improve the accuracy of F-NGBM $(1,1)$. Furthermore, the proposed model can be applied in many other industries with the high fluctuation data to forecast the performances.

\section{Conflict of Interests}

The authors declare that there is no conflict of interests regarding the publication of this paper.

\section{Acknowledgments}

The authors are grateful to the editors and the anonymous reviewers for their helpful and constructive comments and suggestions in editing this paper.

\section{References}

[1] J. L. Deng, Grey Prediction and Decision, Huazhong University of Science and Technology, Wuhan, China, 1986.

[2] J. L. Deng, "Solution of grey differential equation for GM $(1,1 \mid \tau$, r) in matrix train," Journal of Grey System, vol. 13, pp. 105-110, 2002.

[3] J. L. Deng, “Control problems of grey systems," Systems \& Control Letters, vol. 1, no. 5, pp. 288-294, 1982.

[4] Y. Lin and S. Liu, "A historical introduction to grey systems theory," in Proceedings of the IEEE International Conference on Systems, Man and Cybernetics (SMC '04), vol. 3, pp. 2403-2408, IEEE, October 2004.

[5] S. Liu, F. Jeffrey, and Y. Yang, "A brief introduction to grey systems theory," Grey Systems: Theory and Application, no. 2, pp. 89-104, 2012.
[6] Y. L. Huang and Y. H. Lee, "Accurately forecasting model for the stochastic volatility data in tourism demand," Modern Economy, vol. 2, no. 5, pp. 823-829, 2011.

[7] F.-L. Chu, "Forecasting tourism demand in Asian-Pacific countries," Annals of Tourism Research, vol. 25, no. 3, pp. 597-615, 1998.

[8] F. Jiang and K. Lei, "Grey prediction of port cargo throughput based on GM (1, 1, a) model," Logistics Technology, vol. 9, pp. 68-70, 2009.

[9] Z. J. Guo, X. Q. Song, and J. Ye, "A Verhulst model on time series error corrected for port throughput forecasting," Journal of the Eastern Asia Society for Transportation Studies, vol. 6, pp. 881891, 2005.

[10] I. J. Lu, C. Lewis, and S. J. Lin, "The forecast of motor vehicle, energy demand and $\mathrm{CO}_{2}$ emission from Taiwan's road transportation sector," Energy Policy, vol. 37, no. 8, pp. 29522961, 2009.

[11] E. Kayacan, B. Ulutas, and O. Kaynak, "Grey system theorybased models in time series prediction," Expert Systems with Applications, vol. 37, no. 2, pp. 1784-1789, 2010.

[12] M. Askari and H. Askari, "Time series grey system predictionbased models: gold price forecasting," Trends in Applied Sciences Research, vol. 6, no. 11, pp. 1287-1292, 2011.

[13] Y.-F. Wang, "Predicting stock price using fuzzy grey prediction system," Expert Systems with Applications, vol. 22, no. 1, pp. 3338, 2002.

[14] C.-T. Lin and S.-Y. Yang, "Forecast of the output value of Taiwan's IC industry using the grey forecasting model," International Journal of Computer Applications in Technology, vol. 19, no. 1, pp. 23-27, 2004.

[15] L.-C. Hsu, "Applying the Grey prediction model to the global integrated circuit industry," Technological Forecasting and Social Change, vol. 70, no. 6, pp. 563-574, 2003.

[16] L.-C. Hsu, "A genetic algorithm based nonlinear grey Bernoulli model for output forecasting in integrated circuit industry," Expert Systems with Applications, vol. 37, no. 6, pp. 4318-4323, 2010.

[17] L.-C. Hsu, "Using improved grey forecasting models to forecast the output of opto-electronics industry," Expert Systems with Applications, vol. 38, no. 11, pp. 13879-13885, 2011.

[18] D.-C. Li, C.-J. Chang, C.-C. Chen, and W.-C. Chen, "Forecasting short-term electricity consumption using the adaptive greybased approach - an Asian case," Omega, vol. 40, no. 6, pp. 767773,2012

[19] C.-C. Hsu and C.-Y. Chen, "Applications of improved grey prediction model for power demand forecasting," Energy Conversion and Management, vol. 44, no. 14, pp. 2241-2249, 2003.

[20] J. Kang and H. Zhao, "Application of improved grey model in long-term load forecasting of power engineering," Systems Engineering Procedia, vol. 3, pp. 85-91, 2012.

[21] Y.-H. Lin, C.-C. Chiu, P.-C. Lee, and Y.-J. Lin, "Applying fuzzy grey modification model on inflow forecasting," Engineering Applications of Artificial Intelligence, vol. 25, no. 4, pp. 734-743, 2012.

[22] Z. X. Wang, Y. G. Dang, and S. F. Liu, "The optimization of background value in GM $(1,1)$ model," Journal of Grey System, vol. 10, no. 2, pp. 69-74, 2007.

[23] C.-H. Wang and L.-C. Hsu, "Using genetic algorithms grey theory to forecast high technology industrial output," Applied Mathematics and Computation, vol. 195, no. 1, pp. 256-263, 2008. 
[24] C.-N. Wang and V.-T. Phan, "Enhancing the accurate of grey prediction for GDP growth rate in Vietnam," in Proceedings of the International Symposium on Computer, Consumer and Control (IS3C '14), pp. 1137-1139, IEEE, Taichung, Taiwan, June 2014.

[25] S. F. Liu and Y. Lin, Grey Information: Theory and Practical Applications, Springer, London, UK, 2006.

[26] S. Dong, K. Chi, Q. Y. Zhang, and X. D. Zhang, “The application of a Grey Markov Model to forecasting annual maximum water levels at hydrological stations," Journal of Ocean University of China, vol. 11, no. 1, pp. 13-17, 2012.

[27] Y.-T. Hsu, M.-C. Liu, J. Yeh, and H.-F. Hung, "Forecasting the turning time of stock market based on Markov-Fourier grey model," Expert Systems with Applications, vol. 36, no. 4, pp. 8597-8603, 2009.

[28] C.-I. Chen, "Application of the novel nonlinear grey Bernoulli model for forecasting unemployment rate," Chaos, Solitons and Fractals, vol. 37, no. 1, pp. 278-287, 2008.

[29] C.-I. Chen, H. L. Chen, and S.-P. Chen, "Forecasting of foreign exchange rates of Taiwan's major trading partners by novel nonlinear Grey Bernoulli model NGBM $(1,1)$," Communications in Nonlinear Science and Numerical Simulation, vol. 13, no. 6, pp. 1194-1204, 2008.

[30] S. F. Liu, Y. G. Dang, and Z. G. Fang, The Theory of Grey System and Its Applications, Science Publishing, Beijing, China, 2004.

[31] J. Zhou, R. Fang, Y. Li, Y. Zhang, and B. Peng, "Parameter optimization of nonlinear grey Bernoulli model using particle swarm optimization," Applied Mathematics and Computation, vol. 207, no. 2, pp. 292-299, 2009.

[32] C.-I. Chen, P.-H. Hsin, and C.-S. Wu, “Forecasting Taiwan's major stock indices by the Nash nonlinear grey Bernoulli model," Expert Systems with Applications, vol. 37, no. 12, pp. 7557-7562, 2010.

[33] Z.-X. Wang, K. W. Hipel, Q. Wang, and S.-W. He, "An optimized $\operatorname{NGBM}(1,1)$ model for forecasting the qualified discharge rate of industrial wastewater in China," Applied Mathematical Modelling, vol. 35, no. 12, pp. 5524-5532, 2011.

[34] Z.-X. Wang, "An optimized Nash nonlinear grey Bernoulli model for forecasting the main economic indices of high technology enterprises in China," Computers \& Industrial Engineering, vol. 64, no. 3, pp. 780-787, 2013.

[35] C. N. Wang and V. T. Phan, "An improvement the accuracy of grey forecasting model for cargo throughput in international commercial ports of Kaohsiung," International Journal of Business and Economics Research, vol. 3, no. 1, pp. 1-5, 2014.

[36] S. Makridakis, "Accuracy measures: theoretical and practical concerns," International Journal of Forecasting, vol. 9, no. 4, pp. 527-529, 1993.

[37] Website of Kitco, http://www.kitco.com/gold.londonfix.html. 


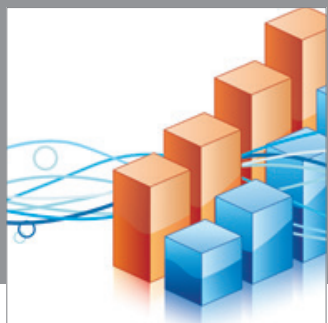

Advances in

Operations Research

mansans

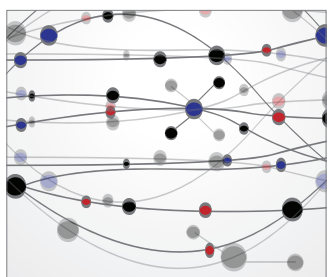

The Scientific World Journal
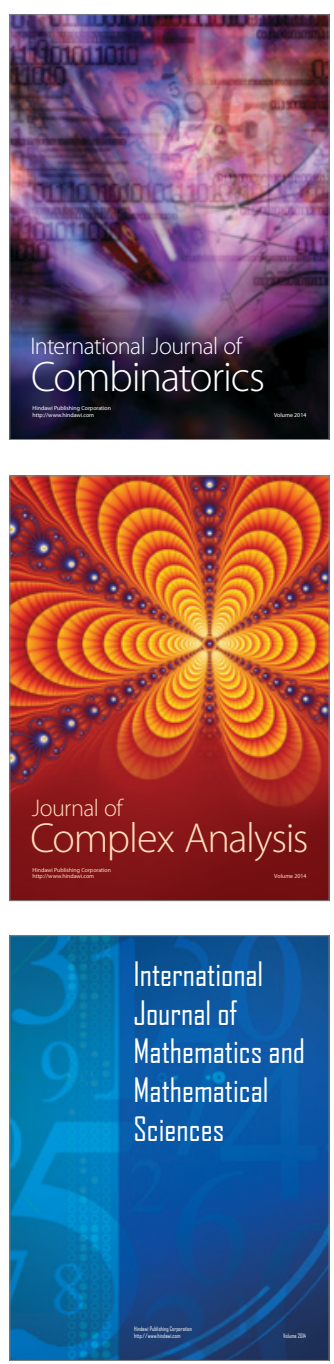
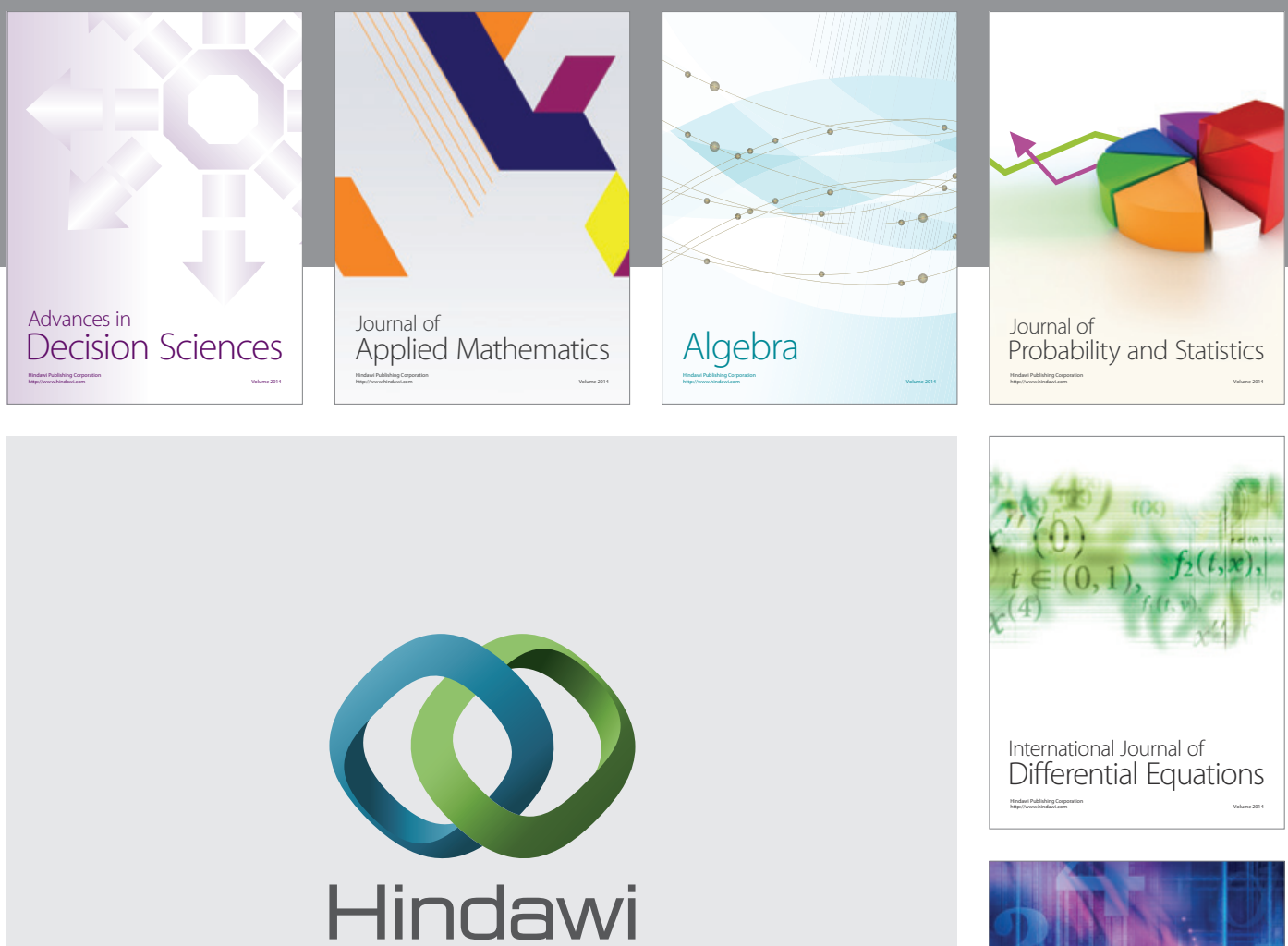

Submit your manuscripts at http://www.hindawi.com
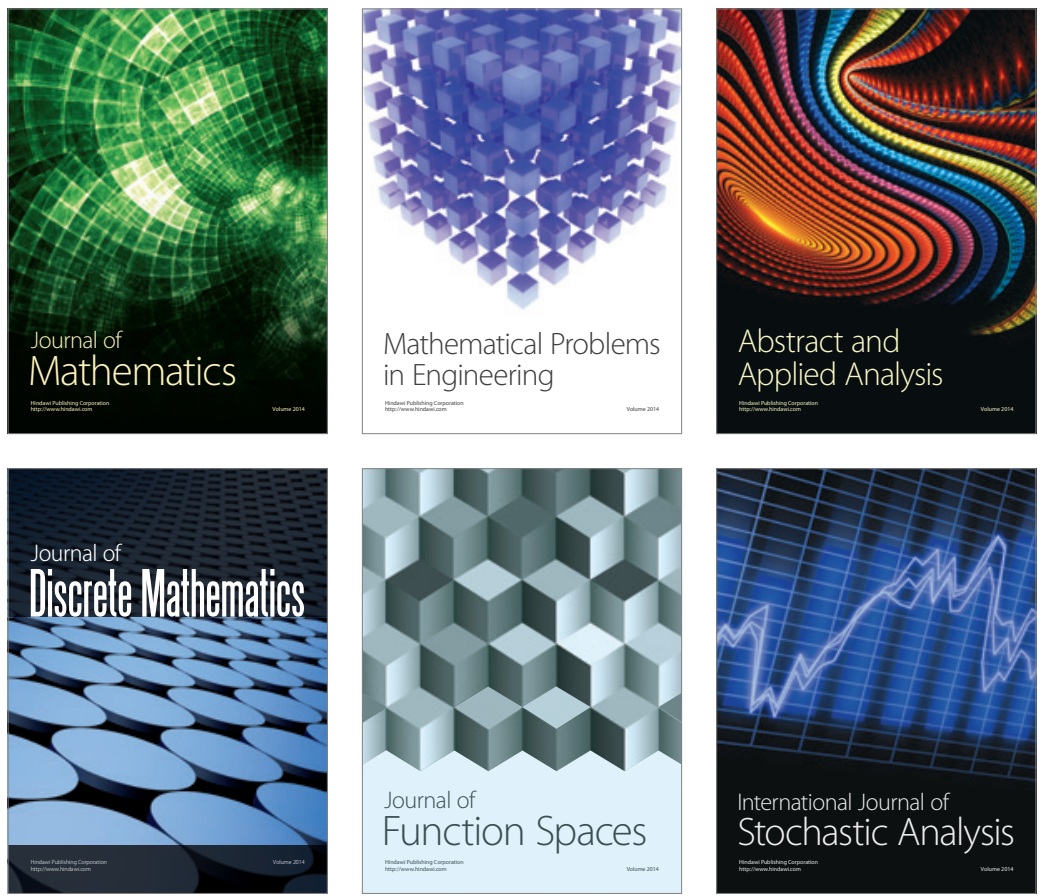

Journal of

Function Spaces

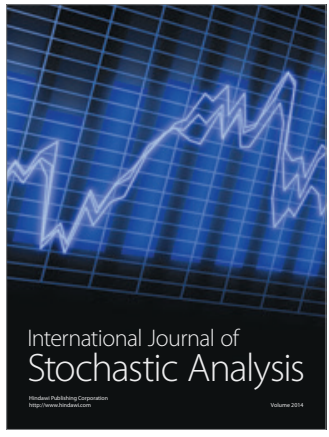

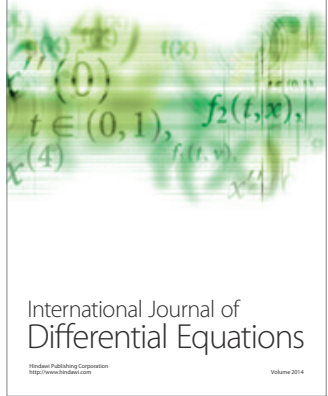
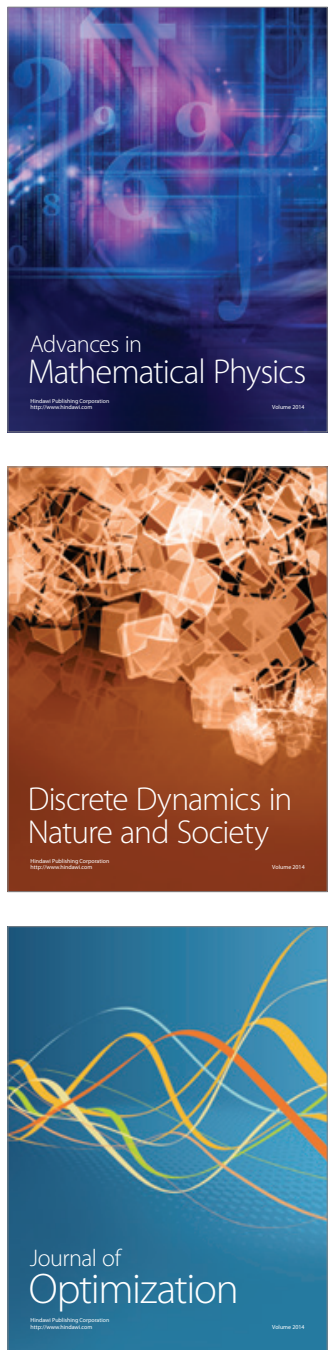